\title{
Resposta de Cosmopolites sordidus (Germar) (Coleoptera, Curculionidae) aos voláteis da planta hospedeira e de adultos coespecíficos em olfatômetro
}

\author{
Fernando A. C. de Mendonça ${ }^{1}$ \\ Evaldo F. Vilela ${ }^{2}$ \\ Álvaro E. Eiras ${ }^{3}$ \\ Antonio E. G. Sant'Ana ${ }^{4}$
}

\begin{abstract}
Response of Cosmopolites sordidus (Germar) (Coleoptera, Curculionidae) to host volatiles and conspecific in olfactometer. The response of Cosmopolites sordidus (Germar, 1824) to the volatiles of its host banana plant (Musa paradisiaca $\mathrm{cv}$. Prata) and conspecific adults was studied. In olfactometer, the attraction of males and females of $C$. sordidus to rhizome and pseudotem of the banana, fresh or rotting, was observed. The results suggested that the aggregation pheromone is produced by males and that it attracts both sexes, and that there is another pheromone produced by females which attracts males only. The results also suggest that the females are the first responsible for the aggregation of the species, but the male pheromone is mainly responsible for the mass aggregation. Nevertheless, the aggregation phenomenon is a consequence of the mutual action of both pheromones plus the kairomones produced by the banana plant.

KEY WORDS. Cosmopolites sordidus, Banana weevil, agregation pheromone, kairomone, olfactometer
\end{abstract}

O moleque-da-bananeira, Cosmopolites sordidus (Germar, 1824) (Coleoptera, Curculionidae), é considerado como praga-chave da bananeira chegando a reduzir em $30 \%$ a produção (ARLEU \& SILVEIRA-NETO 1984). Suas larvas ao se alimentarem dos tecidos do rizoma constróem galerias e provocam a interrupção da seiva e consequentemente o apodrecimento da planta. A utilização de iscas tóxicas, como medida de controle é muito empregada e constam de pedaços de pseudocaule fendidos ao meio e pulverizados com inseticida químico ou Beauveria bassiana (Bals.) Vuill. (RAGA 1994). Entretanto, não existem meios de potencializá-las, associando-as, por exemplo, à armadilha, e dispensando o uso de agrotóxicos.

1) Departamento de Zoologia, Universidade Federal do Paraná. Caixa Postal 19020, 81531-990 Curitiba, Paraná, Brasil.

2) Departamento de Biologia Animal, Universidade Federal de Viçosa, 36571-000, Viçosa, Minas Gerais, Brasil.

3) Departamento de Parasitologia, Universidade Federal de Minas Gerais. Belo Horizonte, Minas Gerais, Brasil.

4) Laboratório de Pesquisa em Recursos Naturais, Universidade Federal de Alagoas. Rodovia BR 101, Tabuleiro dos Martins, 57072-970, Maceió, Alagoas, Brasil. 
Dentre os semioquímicos, o feromônio de agregação produzidos pelos machos de $C$. sordidus e os cairomônios produzidos pela planta hospedeira são considerados preponderantes na comunicação química do moleque-da-bananeira (VIANA \& VILELA 1996). O presente trabalho teve como objetivo estudar, por meio de olfatômetro, o comportamento de agregação do C. sordidus. Procurou-se determinar a atividade biológica dos extratos obtidos da coleta dos voláteis dos insetos e da planta hospedeira, envolvidos no comportamento de agregação.

\section{MATERIAL E MÉTODOS}

\section{Obtenção dos Insetos}

Adultos de C. sordidus foram capturados em plantação de banana (cv. Prata) no município de Marechal Deodoro, Alagoas e sexados (LoNGORIA 1968). Os insetos foram colocados em potes plásticos de 3,0 L; alimentados com pedaços de pseudocaule e mantidos a $25 \pm 1^{\circ} \mathrm{C}, 75 \pm 5 \%$ UR e fotofase de 12 horas.

\section{Coleta dos Voláteis}

Separadamente foram coletados os voláteis de $800 \mathrm{~g}$ de rizoma e de pseudocaule em diferentes estados de conservação (fresco e fermentado). Cada material foi depositado em um balão de 2,0 L e colocado em um sistema de aeração (Fig. 1), com um fluxo de ar de $400 \mathrm{~mL} / \mathrm{s}$ por 144 horas. $\mathrm{O}$ ar era purificado em uma coluna de vidro contendo carvão ativado granulado (20-40 mesh) mais peneira molecular $5 \AA$. Os voláteis liberados foram carreados e ficavam adsorvidos em uma coluna similar contendo $500 \mathrm{mg}$ de carvão ativado granulado. A cada 72 horas a coluna era trocada, obtendo os extratos frescos (das primeiras 72 horas) e fermentados (das 72 horas subseqüentes). As colunas contendo os voláteis foram eluídas com $13 \mathrm{~mL}$ de diclorometano. Sessenta insetos machos e 60 fềmeas foram colocados separadamente em um balão de três bocas $(250 \mathrm{~mL})$ e conectado em outro sistema de aeração (Fig. 2). Similarmente, os voláteis emitidos pelos insetos foram carreados por uma corrente de ar purificado e umedecido por $48 \mathrm{~h}$ e ficaram adsorvidos em colunas contendo carvão ativado granulado $(500 \mathrm{mg})$, sendo a seguir eluídos com $13 \mathrm{~mL}$ de diclorometano.

\section{Testes comportamentais.}

Foi utilizado um olfatômetro (Fig. 3) com fluxo laminar de ar, composto por uma arena retangular $(52 \mathrm{~cm} \times 44 \mathrm{~cm} \times 13 \mathrm{~cm})$, revestida por placas de acrílico. $\mathrm{O}$ fundo e a tampa da arena foram quadriculados, com o objetivo de se determinar uma área útil de observação e promover resposta motora dos insetos. A corrente de ar produzida por um pequeno ventilador entrava no interior da arena na forma de um tênue fluxo laminar e constante $(15 \mathrm{~cm} / \mathrm{s})$ rente à base da arena. Os insetos foram colocados no centro da arena, enquanto a fonte de estímulo estava $20 \mathrm{~cm}$ acima destes contra a corrente de ar. Deste modo os insetos respondiam aos estímulos propostos.

Usou-se nos testes doses crescentes de 10, 100, 1.000 e $10.000 \mu \mathrm{l}$, com 15 repetições, sendo utilizado um inseto para cada repetição. A análise estatística foi feita por meio do teste $X^{2}$. Os insetos usados foram individualizados em potes plásticos um dia antes de cada bioensaio. Em nenhuma ocasião um mesmo inseto foi utilizado em dias consecutivos. Os bioensaios tinham início a partir da quinta 

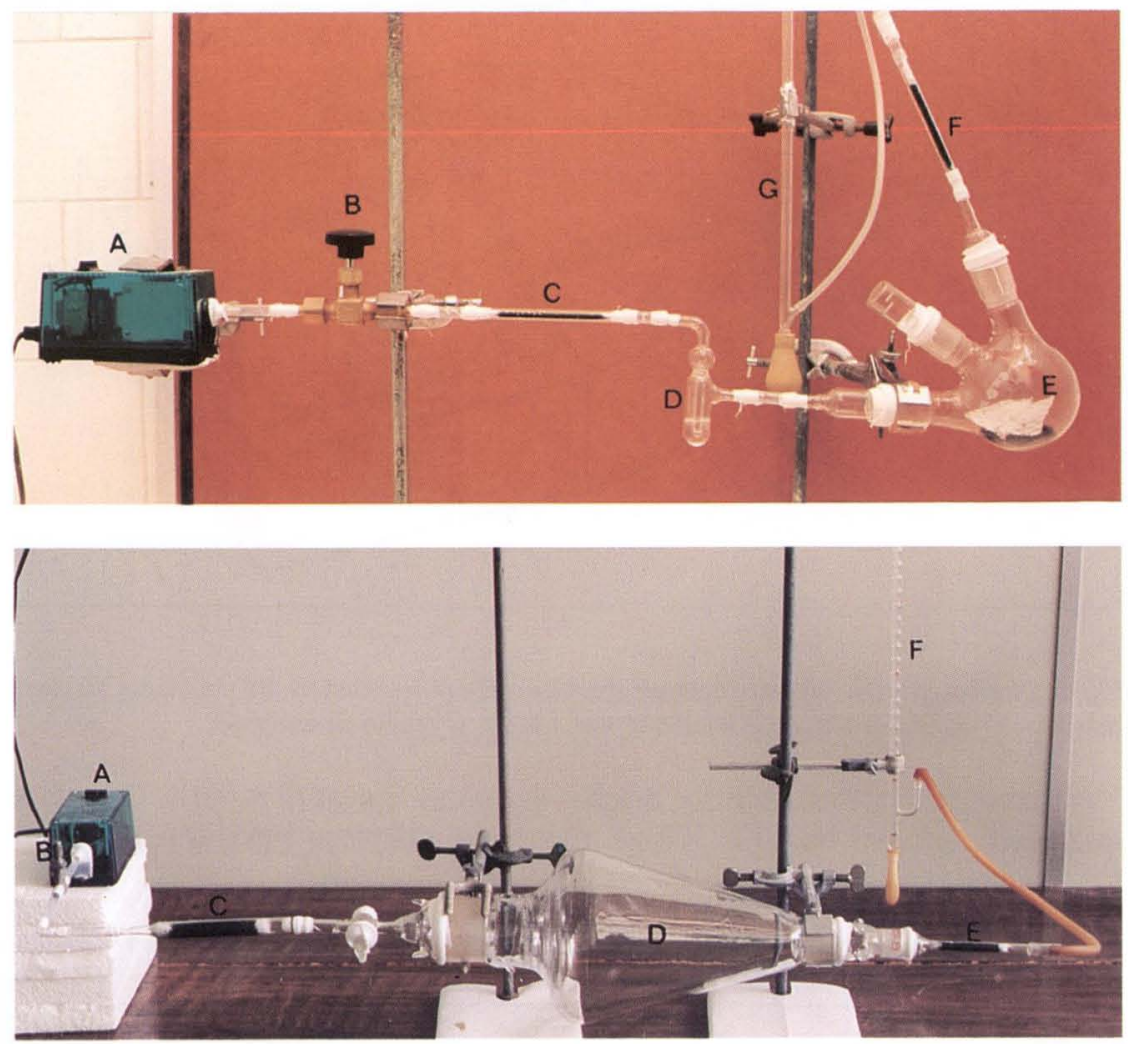

Figs. 1-2. Sistemas de aeração. (1) Utilizado na coleta de voláteis de rizoma e pseudocaule de M. paradisiaca: (A) compressor de ar, (B) válvula de controle de fluxo, (C) filtro de carvão ativado mais peneira molecular, (D) balão (câmara de aeração), (E) coletor de cairomônio, $(F)$ fluxômetro; (2) Utilizado na coleta de feromônio de C. sordidus: (A) compressor de ar, (B) válvula de controle de fluxo, (C) filtro de carvão ativado mais peneira molecular, (D) borbulhador, $(E)$ balão de três bocas (câmara de aeração), ( $F$ ) coletor de feromônio, ( $G$ ) fluxômetro.

hora da escotofase. Considerou-se como resposta positiva $(+)$ o direcionamento dos insetos até a fonte de estímulo e resposta negativa (-) o direcionamento para qualquer outro ponto da arena. Assim que os insetos eram colocados na arena, acionava-se um cronômetro, que era desligado quando cada inseto começava a se movimentar. Caso não se locomovessem ao final de cinco minutos, os insetos eram retirados da arena e considerados como se não respondessem aos tratamentos. Os insetos foram observados individualmente.

\section{RESULTADOS E DISCUSSÃO}

Utilizando-se a dose de $1.000 \mu \mathrm{l}$, observou-se diferença significativa $(\mathrm{P}<$ $0,01)$ para as fêmeas atraídas ao extrato de rizoma fermentado e de machos; enquanto que os machos não tiveram o mesmo comportamento. Na dose de $10.000 \mu \mathrm{l}$ todos os extratos, com exceção do extrato de fêmeas (atrativo apenas aos machos), 


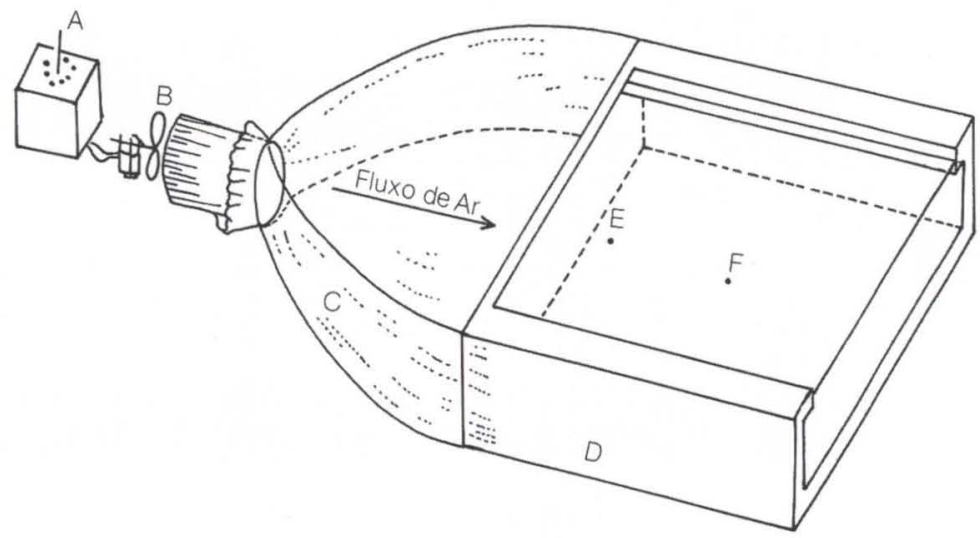

Fig. 3. Olfatômetro: $(A)$ reostato (controle da velocidade do ventilador), $(B)$ ventilador, $(C)$ saco plástico, (D) arena, (E) local de liberação dos insetos, $(F)$ fonte de estímulo.

mostraram-se atrativos tanto aos machos quanto às fêmeas $(\mathrm{P}<0,01)$. Quando se utilizaram as doses de 10 e $100 \mu \mathrm{l}$, não houve diferença significativa entre os tratamentos (Tab. I).

Tabela I. Percentual de respostas de adultos de Cosmopolites sordidus às diferentes doses de extratos de Musa paradisiaca (rizoma e pseudacaule) e de adultos coespecificos.

\begin{tabular}{|c|c|c|c|c|c|}
\hline \multirow{2}{*}{ Extrato } & \multirow{2}{*}{$\begin{array}{l}\text { Inseto teste } \\
\quad(N=15)\end{array}$} & \multicolumn{4}{|c|}{ Doses $(\mu \mathrm{l})$} \\
\hline & & 10 & $10^{2}$ & $10^{3}$ & $10^{4}$ \\
\hline \multirow[t]{2}{*}{ Rizoma fresco } & Macho & 0,0 & 0,0 & 13,30 & 40,00 \\
\hline & Fêmea & 6,6 & 13,3 & 6,60 & $40,00^{*}$ \\
\hline \multirow[t]{2}{*}{ Rizoma fermentado } & Macho & 0,0 & 0,0 & 13,30 & $46,60^{*}$ \\
\hline & Fêmea & 0,0 & 6,6 & 40,00 * & 46,60 * \\
\hline \multirow[t]{2}{*}{ Pseudocaule fresco } & Macho & 0,0 & 0,0 & 13,30 & $40,00^{*}$ \\
\hline & Fêmea & 6,6 & 13,3 & 6,60 & $40,00^{*}$ \\
\hline \multirow[t]{2}{*}{ Pseudocaule fermentado } & Macho & 0,0 & 0,0 & 13,30 & $40,00^{*}$ \\
\hline & Fêmea & 13,3 & 6,6 & 6,60 & $46,60^{*}$ \\
\hline \multirow[t]{2}{*}{ Feromônio de machos } & Macho & 6,6 & 0,0 & 6,60 & $66,67^{*}$ \\
\hline & Fêmea & 6,6 & 6,6 & $33,33^{*}$ & 73,33 \\
\hline \multirow[t]{2}{*}{ Feromônio de fêmeas } & Macho & 0,0 & 0,0 & 13,30 & $40,00^{*}$ \\
\hline & Fêmea & 0,0 & 0,0 & 0,00 & 13,30 \\
\hline \multirow[t]{2}{*}{ Controle (Diclorometano) } & Macho & 0,0 & 0,0 & 0,00 & 6,60 \\
\hline & Fẽmea & 0.0 & 0,0 & 0,00 & 0,00 \\
\hline
\end{tabular}

$\left(^{*}\right)$ Diferença estatística $(P<0,01)$ pelo teste $X 2$.

Os machos responderam aos extratos apenas na dose de $10.000 \mu \mathrm{l}$, enquanto as fêmeas começaram a responder em uma dose dez vezes inferior à requerida pelos machos (Tab. I). Sugere-se, portanto, que exista uma diferença qualitativa ou quantitativa nas sensilas responsáveis pela percepção dos odores do hospedeiro e de coespecíficos, entre os sexos de C. sordidus. As fêmeas perceberam mais facilmente os voláteis da bananeira que os machos, sugerindo que a orientação até o hospedeiro esteja associada não apenas com a fonte de alimento, mas também a 
sítios de oviposição, o que difere dos resultados de BUDENBERG et al. (1993b). No entanto os resultados estão de acordo com CuILlé (1950) e PAVIS (1988), que sugeriram que as substâncias presentes no rizoma também induzem à oviposição e que a atração de machos e fêmeas pelo rizoma resulta, consequentemente, em acasalamentos bem sucedidos.

Este resultado levanta a possibilidade de que sejam as fêmeas o sexo iniciador da agregação de $C$. sordidus, visto que possuem uma sensibilidade nitidamente superior a dos machos, podendo perceber os voláteis do rizoma fermentado a uma distância maior, como ocorre com várias espécies de coleópteros (BIRCH 1984; BORDEN 1984).

BUDENBERG et al. (1993a) observaram em olfatômetro e por meio de eletroantenografia (EAG) a atração de indivíduos de $C$. sordidus pelo extrato de bananeira, porém, não verificaram a atração dos mesmos pela mistura sintética dos componentes do rizoma identificados por NDIEGE et al. (1991).

BUDENBERG et al. (1993b) sugerem que, provavelmente, os machos de $C$. sordidus produzem o feromônio de agregação via intestino médio, o que foi evidenciado em olfatômetro e EAG, corroborando deste modo os dados obtidos por VIANA \& VILELA (1996). Observou-se, também, uma tendência dos insetos machos preferirem o extrato dos machos ao das fêmeas, apesar de não ser detectada uma diferença significativa.

De acordo com os resultados, o feromônio liberado pelo machos é comprovadamente mais atrativo que o feromônio liberado pelas fêmeas, evidenciando-se, com isso, ser o responsável pela agregação em massa em torno da fonte de alimento.

O feromônio produzido pela fêmeas, atrativo somente aos machos, já fora detectado por Viana \& Vilela (1996) e BUDENBERG et al. (1993b), que segundo estes, a maior resposta das fêmeas aos extratos poderia ser explicada pelo processo de habituação. No entanto, não foi observado nenhum aumento de resposta dos machos ao extrato do feromônio dos machos ou ao extrato de rizoma fermentado, mesmo estes ficando individualizados em potes plásticos, como foi verificado com as fêmeas.

As fêmeas, pelo fato de serem significativamente mais sensíveis aos extratos da planta hospedeira possuem maior chance de chegar à fonte de alimento e, ao chegarem à mesma, liberam um feromônio que atrairia somente machos. Estes ao chegarem até o local, guiados pelo feromônio das fêmeas, iniciariam a liberação de um outro feromônio, que desta vez atrairia ambos os sexos.

Recentemente, o feromônio de agregação produzido pelos machos de $C$. sordidus foi identificado e sintetizado como sendo um diastereoisômero (1 $\left.\mathrm{S}^{*}, 3 \mathrm{R}^{*}, 5 \mathrm{R}^{*}, 7 \mathrm{~S}^{*}\right)$ 2,8-dioxa 1-etil 3,5,7-trimetil biciclo[3.2.1] octano, que recebeu o nome comum de sordidina (BEAUHAIRE et al. 1995), por meio de testes de campo foi confirmada sua atividade biológica (NDIEGE et al. 1996). 


\section{REFERÊNCIAS BIBLIOGRÁFICAS}

ARLEU, R.J. \& S. SilveIRA-NETO. 1984. Broca da bananeira Cosmopolites sordidus (Germar, 1824) (Coleoptera: Curculionidae). Turrialba 34: 359-367.

Beauhaire, J.; P.H. Ducrot; C. Malosse; D. Rochat; I.O. Ndiege \& D.O. OTIENO. 1995. Identification and synthesis of sordidin, a male pheromone emitted by Cosmopolites sordidus. Tetrahedron Lett. 36: 1043-1046.

BIRCH, M.C. 1984. Aggregation in bark beetle, p.331-353. In: W.J. BELL \& R.T. CARDÉ. Chemical Ecology of Insects. New York, Chapmam and Hall, ???p.

BORDEN, J.H.B. 1984. Semiochemical-mediated aggregation and dispersion in the Coleoptera, p.123-1449. In: T. LEWIS. Insect communication. London, Academic Press.

BUdENBERG, W.J.; I.O. Ndiege; F.W. KARAGO \& B.S. HANSON. 1993a. Behavior and electrophysiological response of the banana weevil, Cosmopolites sordidus to host plant. Jour. Chem. Ecol. 19: 267-278.

1993b. Evidence for a volatile male-produced pheromone in the banana weevil, Cosmopolites sordidus. Jour. Chem. Ecol. 19: 1905-1916.

CuILLÉ, J. 1950. Recherches sur le charançon du bananier Cosmopolites sordidus, Germ., Momographie de l'insecte et recherches de ses chimiotropisme. Paris, Institut des Fruits et Agrume Coloniaux, Ser. tech. 4, $225 p$.

LONGORIA, A.G.G. 1968. Diferencias sexuales en la morfologia externa de Cosmopolites sordidus Germar (Coleoptera: Curculionidae). Ciências, série 4, Habana: 1-11.

Ndiege, I.O.; W.J. Budenberg; W. Lwander \& A. Hassanali. 1991. Volatile components of banana pseutem of a cultivar susceptible to the banana weevil. Phytochemistry 30: 3929-3930.

Ndiege, I.O.; S. Jayaraman; A.C. Oehlschlager; L. GonZalez; D. Alpizar $\&$ M. FALLAS. 1996. Convenient synthesis and field activity of a male-produced aggregation pheromone of Cosmopolites sordidus. Naturwissenschaften 83: 280-282.

PAVIS, C. 1988. Quelques aspects comportementaux chez le charançon du bananier Cosmopolites sordidus Germar (Coleoptera: Curculionidae). Montpellier, International Net Work for the Improvement of Banana and Plantain, 58-61.

Raga, A. 1994. Principais pragas da Bananeira, p.51-57. In: ?. BATISTA FILHO \& A. RAgA (Ed.). Anais do I Ciclo de Palestra sobre a Cultura da Bananeira. Campinas, Editora, ???p.

ViANA, A.M.M. \& E.F. VILELA. 1996. Comportamento corte e e acasalamento de Cosmopolites sordidus (Coleoptera: Curculionidae) An. Soc. Entomol. Brasil 25: 347-350.

Recebido em 29.I.1999; aceito em 17.XI.1999. 